\section{Is optical coherence tomography an effective device for evaluation of tear film meniscus in patients with acne rosacea?}

FC Eroglu ${ }^{1}$, A Karalezli ${ }^{2}$ and R Dursun ${ }^{3}$

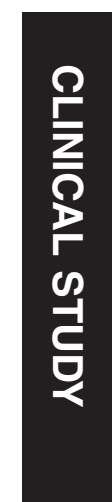

Introduction

Acne rosacea is considered as a primarily chronic skin disease characterized by recurrent episodes of facial erythema, telangiectases, papules, and pustules, but it may also affect the eyes. ${ }^{1}$ The pathogenesis of the acne rosacea remain unknown. ${ }^{1,2}$ The incidence of ocular involvement varies between different studies. ${ }^{2-4}$ The most frequent ocular manifestations include blepharitis, meibomitis, keratoconjuntivitis sicca, lid margin telangiectasia, marginal corneal ulcers, and vascularization. Foreign body sensation, photophobia, itching, redness, dryness, burning or tearing are some of the symptoms seen in ocular rosacea. ${ }^{3-5}$ In the several studies, the authors reported that meibomian glands dysfunction may lead to an abnormal lipid composition of the tear film, and so dry eye is common in the ocular rosacea. ${ }^{6-9}$

To date there is no gold standard diagnostic test for dry eye in practice. Optical coherence tomography (OCT) is a noninvasive, highresolution imaging technique that employs low-coherence interferometry to provide detailed cross-sectional images of biological tissues. ${ }^{10}$ OCT has been used to evaluate the anterior segment, and it is increasingly being recognized as a noninvasive and precise alternative for measuring tear meniscus. ${ }^{11-13}$ Tear meniscus height (TMH), tear meniscus depth (TMD) and tear meniscus cross-sectional area (TMA) measurements by using OCT are good indicators of tear meniscus. ${ }^{14}$ Although a variety of recent studies suggest that measurement of tear mensicus by using OCT has potential for diagnosis and monitoring in different types of dry eye, there is a paucity of research on concerning with diagnostic
${ }^{1}$ Department of Ophthalmology, Sereflikochisar State Hospital, Ankara, Turkey

${ }^{2}$ Department of Ophthalmology, Baskent University School of Medicine, Konya, Turkey

${ }^{3}$ Department of Dermatology, Baskent University School of Medicine, Konya, Turkey

Correspondence: Dr FC Eroglu, Department of Ophthalmology, Sereflikochisar State Hospital, 06950 Sereflikochisar, Ankara, Turkey Tel: +90 312 6871754; Fax: +90 3126865009 . E-mail: dr_fatoscorak@ hotmail.com

Received: 8 April 2015 Accepted in revised form: 13 November 2015 Published online: 15 January 2016 
significance of OCT in dry eye with systemic disorders. ${ }^{15-17}$ A thorough search of the literature identified no studies of OCT findings in patients with acne rosacea. Therefore, in this study, we intended to measure the TMH, TMD, and TMA by using Fourierdomain OCT (FD-OCT) in patients with acne rosacea and to determine the relationship between tear film break-up tests (TBUT), Schirmer test, and OCT values and to compare them with that of healthy controls.

\section{Methods}

This prospective case-control clinical study was performed between Nov 2012 and Dec 2013. Patients were divided into two groups. Group 1 included 104 eyes of 104 patients (44 males and 60 females) with acne rosacea who were referred from the Dermatology Department for ophthalmological examination. Group 2 included 104 eyes of 104 healthy sex and age-matched volunteers (44 males and 60 females) presenting to Ophthalmology Department for routine eye examination. All patients were initially seen for a dermatologic history and examination. The diagnosis of acne rosacea was based on the standard classification system reported by the Expert National Rosacea Society Committee. ${ }^{18}$

The study was approved by the local ethics committee of Baskent University School of Medicine and conducted in accordance with the tenets of the Declaration of Helsinki. The detailed written informed consent form was obtained each participant. Patients were excluded if they wore contact lens, used any topical ophthalmologic medication or systemic immunosuppressive (including corticosteroid) therapy within the previous 1 year, or had a history of ocular surface disorder (Sjogren's syndrome, ocular cicatricial pemphigoid or any other type of conjunctivitis or keratitis), eyelid problems (ectropion, entropion, lagophthalmos, or pitosis), previous ocular surgery, or ocular injury. Control subjects had no ocular problems except for presbyopia or a refractive error within \pm 3.00 diopters, no history of ocular surgery, no major systemic disease affecting the ocular tissues, and did not wear contact lenses. None of the patients or controls had cigarette and/or alcohol consumption history and were receiving any systemic or topical medication that could affect the tear film or meibomian gland functions.

Subjective ocular symptoms were evaluated with the Ocular Surface Disease Index (OSDI) questionnaire, ${ }^{19}$ which was adopted to Turkish people, made of 12 questions relating to the effect of dry eye on vision-related functioning. ${ }^{20}$ All participants underwent detailed ophthalmic examination, including visual acuity testing, slit-lamp biomicroscopy (particular attention to the lid margins, tarsal and bulbar conjunctiva, and cornea) and dilated fundus examination. In addition, all patients underwent OCT imaging, as well as TBUT and Schirmer's tests. One eye (right eye) per patient was selected for all evaluation. All tests were performed at the same time of the day (between 1000 hours and 1200 hours) in a dimly lit room and patients were instructed not to use any eye drops, including artificial tear preparations, $2 \mathrm{~h}$ before testing.

Before TBUT and Schirmer, OCT measurements were undertaken by the certified technician who masked to clinical parameters of the participitans using FD-OCT device ( $\lambda=840 \mathrm{~nm}, 26000 \mathrm{~A}$-scans $/ \mathrm{s}$, and $5 \mu \mathrm{m}$ axial resolution to depth of $2.8 \mathrm{~mm}$ ), equipped with a the cornea/anterior module-short (CAM-S) to obtain anterior segment images tests. The TMH, TMD, and TMA of all participants were measured. The participants were instructed to rest their foreheads against the headrest, their chins on the chin rest, and to gaze at the built-in targets and blink normally to evenly distribute tear film and minimize ocular surface dehydration. Three consecutive scans were performed during each measurement, each after a blink, with a scanning interval of 3-5 s. The results of TMH, TMD, and TMA were analyzed by two masked physicians (FCE ang AK) using the Optovue RTVue software version 3.5 (Optovue Inc., Fremont, CA, USA). Inter examiner reproducibility of the tear meniscus measurements were assessed by measuring the interclass correlation coefficient (ICC). TMH was defined as the straight line distance between the upper extreme (cornea) and the lower extreme (eyelid) of the tear boundary line; TMD as the vertical distance from the interface of the cornea with the lower eyelid to the tear height line; TMA as triangular area formed by the corneal anterior boundary, anterior boundary of the lower eyelid and anterior borderline of the tear meniscus (Figure 1).

TBUT was performed in all patients, following which a fluorescein strip (Flourescein paper, Haag-Streit AG, Köniz, Switzerland) was placed into the lower conjunctival sac, the patient was asked to blink several times and then look straight forward without blinking. The tear film was observed under cobalt blue-filtered light of the slit-lamp biomicroscope and the length of time from fluorescein application to appearance of the first break in the tear film was recorded in seconds. The average of three measurements was accepted as the TBUT.

Schirmer I test without anesthesia was performed by a Schirmer-Plus strip (Whatmann, Maidstone, UK) over the lower lid margin at the junction of the middle and lateral one-third of the eye lid. The patient was asked to close her/his eyes during the course of the test. The distance of wetness in millimeters in the paper after 5 min was recorded as the Schirmer I test value. Subjects who met a following criteria were considered as dry eye: $>10.4$ score 

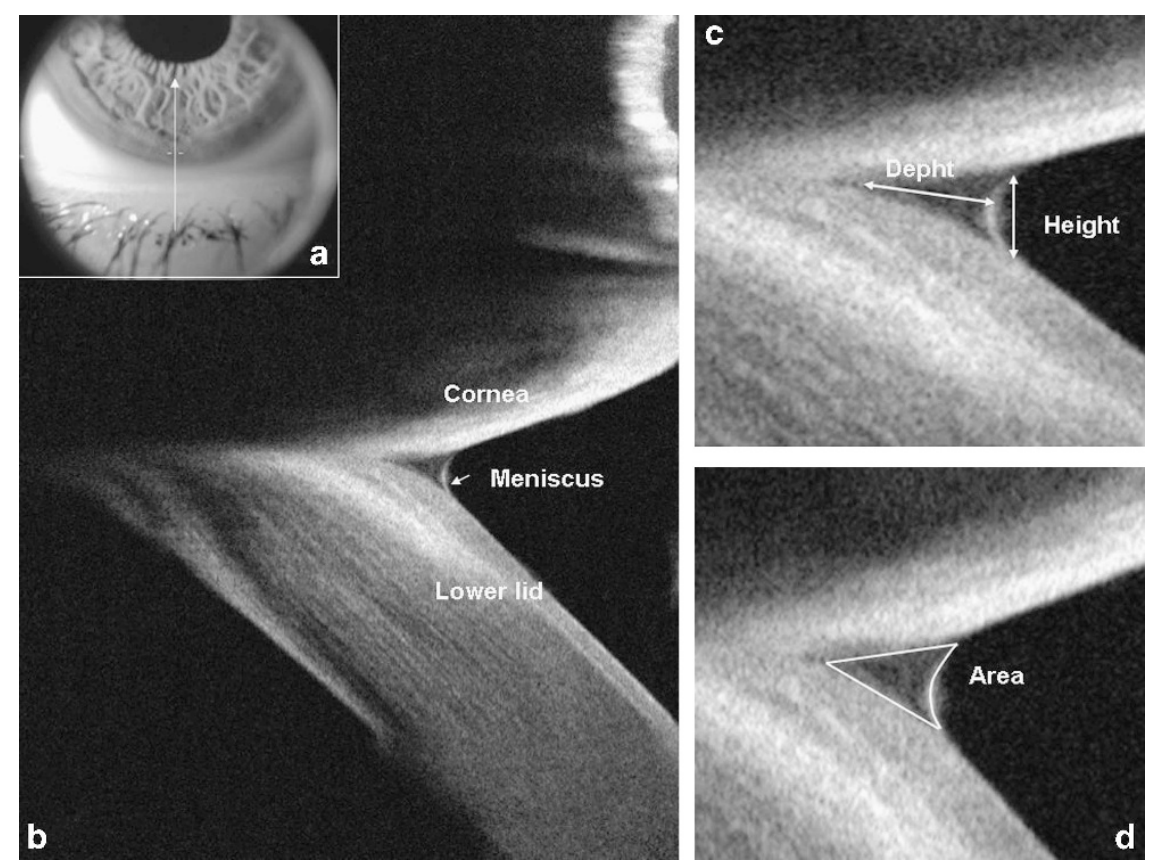

Figure 1 The results of image acquisition by Fourier-domain optical coherence tomography. (a) Video imaging illustrating the vertical scan path (white arrow path) of the optical coherence tomography beam used to image the tear meniscus. The length of scanning line is $3 \mathrm{~mm}$ and scanning direction is $90^{\circ}-270^{\circ}$. Scanning started at the $60^{\prime}$ clock position of the cornea (b) An optical coherence tomography vertical line scan cross-sectional image of the lower tear meniscus. (c) and (d) Caliper measurement protocol.

on OSDI, tear secretion deficiency in the Schirmer test $(10 \mathrm{~mm})$, and TBUT $<10 \mathrm{~s}$.

Statistical analysis was performed using the statistical package SPSS version 15.0 (SPSS Inc., Chicago, IL, USA, 2006). Descriptive data were calculated as the mean \pm SD. Assessment of normality of distribution was performed with Shapiro-Wilk tests. Data were analyzed by MannWhitney $U$-test. The categorical variables between two groups were analyzed using the $\chi^{2}$ and Student's $t$-tests. Correlation was evaluated using Spearman's correlation analysis. Spearman's correlation coefficient $(R)$ value $>0.5$ was considered significant. The area under the receiver operating characteristic (ROC) curve was determined using a logistic regression model. Intraindividual variation in OCT measurements was defined as the repeatability of tear meniscus measurements with OCT. Interindividual variation was defined as dispersion of data distribution between subjects. Reproducibility and repeatability of OCT measurements were evaluated with the ICC. $P$-value $<0.05$ was considered statistically significant.

\section{Results}

The mean age was $38.37 \pm 12.37$ (range, 18-68) in group 1 and $38.24 \pm 11.53$ (range, 19-66) in group 2. There was no statistically significant difference in age between groups $(P=0.38)$. As shown in Table 1 , the mean values for each
Table 1 Clinical examination results of acne rosacea patients and control subjects

\begin{tabular}{lccc}
\hline & $\begin{array}{c}\text { Rosacea group } \\
\text { Mean } \pm S D\end{array}$ & $\begin{array}{c}\text { Control subjects } \\
\text { Mean } \pm S D\end{array}$ & P-value \\
\hline OSDI scores & $23.19 \pm 9.78$ & $12.23 \pm 9.52$ & 0.000 \\
TBUT(s) & $7.25 \pm 3.46$ & $10.08 \pm 2.71$ & 0.000 \\
Schirmer $(\mathrm{mm})$ & $13.27 \pm 6.45$ & $15.55 \pm 4.31$ & 0.003 \\
TMH $(\mu \mathrm{m})$ & $247.70 \pm 22.54$ & $297.33 \pm 48.16$ & 0.000 \\
TMD $(\mu \mathrm{m})$ & $270.87 \pm 25.19$ & $308.32 \pm 37.65$ & 0.014 \\
TMA $\left(\mu \mathrm{m}^{2}\right)$ & $19500 \pm 3256.5$ & $27490 \pm 7724.69$ & 0.000 \\
\hline
\end{tabular}

Abbreviations: OSDI, ocular surface disease index; SD, standard deviation; TBUT, tear film break-up time; TMA, tear meniscus cross-sectional area; TMD, tear meniscus depth; TMH, tear meniscus height.

parameters were significantly lower in acne rosacea patients compared with control subjects $(P<0.001$ for OSDI scores, TBUT, TMH, and TMA; $P=0.003$ for Schirmer tests, $P=0.014$ for TMD).

Correlations between evaluated parameters are shown in Table 2. There was a significant linear positive correlation between TBUT, Schirmer test, TMH, TMD, and TMA values $(P<0.001$, for each set of parameters) and a significant linear negative correlation between OSDI scores and TBUT, Schirmer test, TMH, TMD, and TMA values $(P<0.001$, for each parameters). Correlation coefficient for OSDI and TMH $(R=-0.589)$, OSDI and $\operatorname{TMD}(R=-0.527)$ and OSDI and TMA $(R=-0.613)$, were moderate, however correlation coefficient for OSDI and 
Table 2 Correlation of clinical test results of acne rosacea patients and control subjects (Spearman)

\begin{tabular}{clllll}
\hline & TBUT & Schirmer & TMH & TMD & TMA \\
\hline TMH & & & & & \\
$\mathrm{R}_{2}{ }^{\mathrm{a}}$ & $0.391^{\mathrm{a}}$ & $0.390^{\mathrm{a}}$ & - & - & - \\
$\mathrm{P}^{\mathrm{b}}$ & 0.000 & 0.000 & - & - & - \\
& & & & & \\
$\mathrm{TMD}$ & & & & - & - \\
$\mathrm{R}^{2} \mathrm{a}$ & $0.353^{\mathrm{a}}$ & $0.404^{\mathrm{a}}$ & & - & - \\
$\mathrm{P}$ & 0.000 & 0.000 & 0.000 & - & \\
& & & & & - \\
$\mathrm{TMA}$ & & & & $0.910^{\mathrm{a}}$ & - \\
$\mathrm{R}_{2}{ }^{\mathrm{a}}$ & $0.0 .368^{\mathrm{a}}$ & $0.0 .394^{\mathrm{a}}$ & $0.0 .934^{\mathrm{a}}$ & 0.000 & - \\
$\mathrm{P}$ & 0.000 & 0.000 & 0.000 & & \\
$\mathrm{OSDI}$ & & & & & \\
$\mathrm{R}_{2}$ & $0.267^{\mathrm{a}}$ & $0.312^{\mathrm{a}}$ & $0.589^{\mathrm{a}}$ & $0.527^{\mathrm{a}}$ & $0.613^{\mathrm{a}}$ \\
$\mathrm{P}$ & 0.000 & 0.000 & 0.000 & 0.000 & 0.000 \\
\hline
\end{tabular}

Abbreviations: $p$, significance of correlation; $r$, Spearman's correlation coefficient; TBUT, tear film break-up time; TMA, tear meniscus crosssectional area; TMD, tear meniscus depth; $\mathrm{TMH}$, tear meniscus height. a Significance at the 0.001 level. ${ }^{\mathrm{b}}$ Significance at the 0.05 level.

TBUT $(R=-0.312)$ and OSDI and Schirmer $(R=-0.267)$ were low. There was lack of correlation between OSDI and TBUT, and OSDI and Schirmer tests. Correlation coefficients for TBUT and TMH $(R=0.391)$, TBUT and TMD $(R=0.353)$, TBUT and TMA $(R=0.368)$, were low. Moreover, correlation coefficients for Schirmer and TMH $(R=0.390)$, Schirmer and TMD $(R=0.404)$, Schirmer and TMA $(R=0.394)$, were also low. Also there was lack of correlation between TBUT and tear meniscus, and Schirmer test and tear meniscus parameters. Figure 2 shows scatter plots demonstrating correlation between the variables (OSDI, Schirmer test, TBUT, and tear meniscus parameters) in cases.

We evaluated the diagnostic efficiency of FD-OCT in patients with acne rosacea and compared them with healthy controls. The area under the curve (AUC) was measured using the ROC curve. As showed in Figure 3, the AUC values that were calculated by the ROC technique revealed an acceptable diagnostic efficiency in acne rosacea patients.

The intraobserver ICC was found 0.93 for $\mathrm{TMH}$ (95\% CI), 0.92 for TMD (95\% CI) and 0.96 for TMA (95\% CI) and the interobserver ICC was found 0.94 for TMH (95\% CI), 0.93 for TMD (95\% CI) and 0.96 for TMA (95\% CI) in the rosacea group. The intraobserver ICC was found 0.90 for TMH (95\% CI), 0.93 for TMD (95\% CI) and 0.95 for TMA (95\% CI) and the interobserver ICC was found 0.92 for TMH (95\% CI), 0.94 for TMD (95\% CI) and 0.93 for TMA (95\% CI) in the control subjects. The intraobserver ICC revealed high repeatability and the interobserver ICC revealed high reproducibility for all measurements parameters both acne rosacea and control subjects.

\section{Discussion}

In previous studies, dry eye has been described in the patients with acne rosacea. ${ }^{6-9}$ Quarterman et al found that all patients with cutaneous rosacea had some degree of ocular involvement and TBUT was abnormal in patients with rosacea. Lemb et $\mathrm{al}^{7}$ found that the prevalence of dry eyes were significantly greater in the patients with rosacea. In the study conducted by Zengin et al, Schirmer, and TBUT values in patients with meibomian gland dysfunctions were found to be significantly decreased compared with those of the patients without meibomian gland dysfunction. The authors concluded that there was a positive correlation between tear film abnormalities and meibomian gland dysfunction. ${ }^{8}$ Tear film was evaluated by using traditional diagnostic methods including Schirmer's test, TBUT and fluorescein staining in all of these studies. ${ }^{6-9}$ These conventional methods are currently adopted by most ophthalmologist and commonly used to diagnose dry eye in ophthalmic practice because of they are easy to use, inexpensive, and take a short time to perform. ${ }^{21-23}$ But these methods with invasive nature have some disadvantages such as low reproducibility and repeatability; discomfort; reflex tearing and lack of the control over reflex lacrimation. Moreover, many factors may interfere with the results. ${ }^{22-24}$ Correlations between these various tests and between the tests and signs and symptoms are poor in many studies. ${ }^{25-27}$ For this reason, the availability of a noninvasive, quick, reliable, and comfortable test would be a significant advancement in diagnostic approaches in our practice. Techniques that gather information about the tear film by processing reflected light or images the tear film surface are desirable and representative of the ocular surface. ${ }^{28}$ A number of noninvasive approaches for tear meniscus measurements have been developed in the past several decades, including video assessment, noninvasive interference tear meniscometry, and optical pachymetry. ${ }^{29,30}$ However, these methods have not been used in clinical practise owing to their low accuracy rates and complicated procedures involved. With recent technological advancements, anterior segment OCT (ASOCT) has been described to be a promising non-invasive real-time method for tear meniscus measurement. ${ }^{16,17,31-35}$

Before adopting OCT for management of dry eye, correlations between OCT measurements and conventional tests such as Schirmer's test, TBUT, and fluorescein staining should be evaluated. AS-OCT has been used in various studies to evaluate tear meniscus, tear film, and tear dynamics, and also to determine the 
a

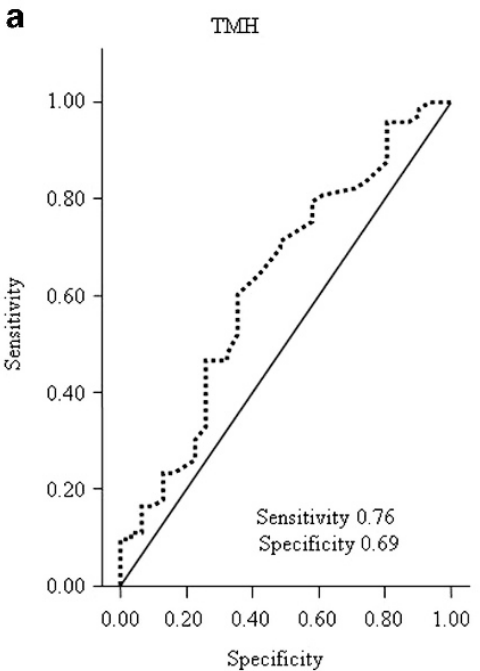

Area under $\mathrm{ROC}$ curve $=0.6280$ b

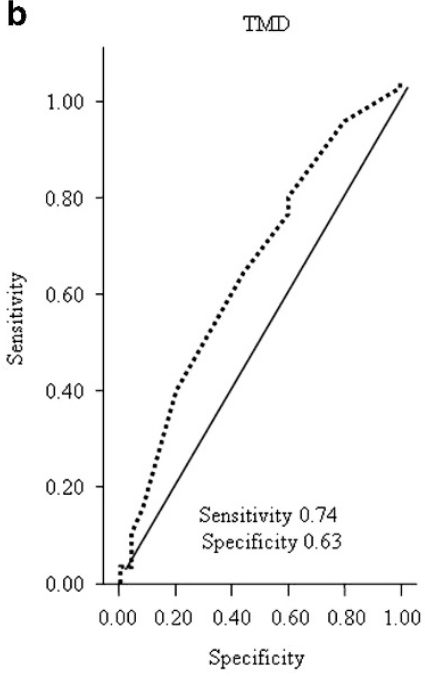

Area under $\mathrm{ROC}$ curve $=0.6010$

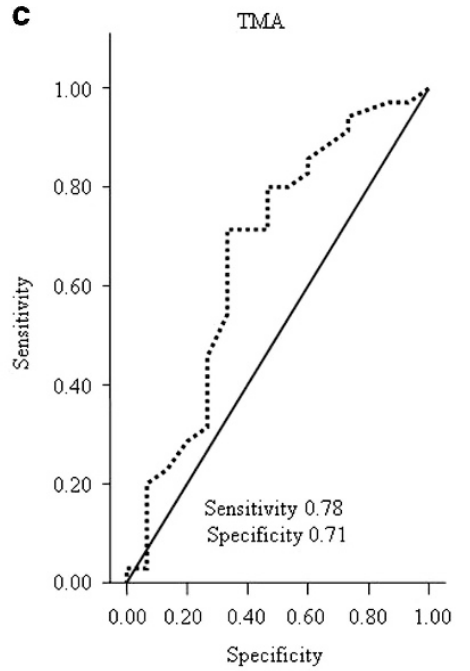

Area under $\mathrm{ROC}$ curve $=0.6660$

Figure 2 Scatter plots demonstrating correlation between Schirmer test results and tears meniscus parameters (a); TBUT and tears meniscus parameters (b); OSDI and and tears meniscus parameters (c).
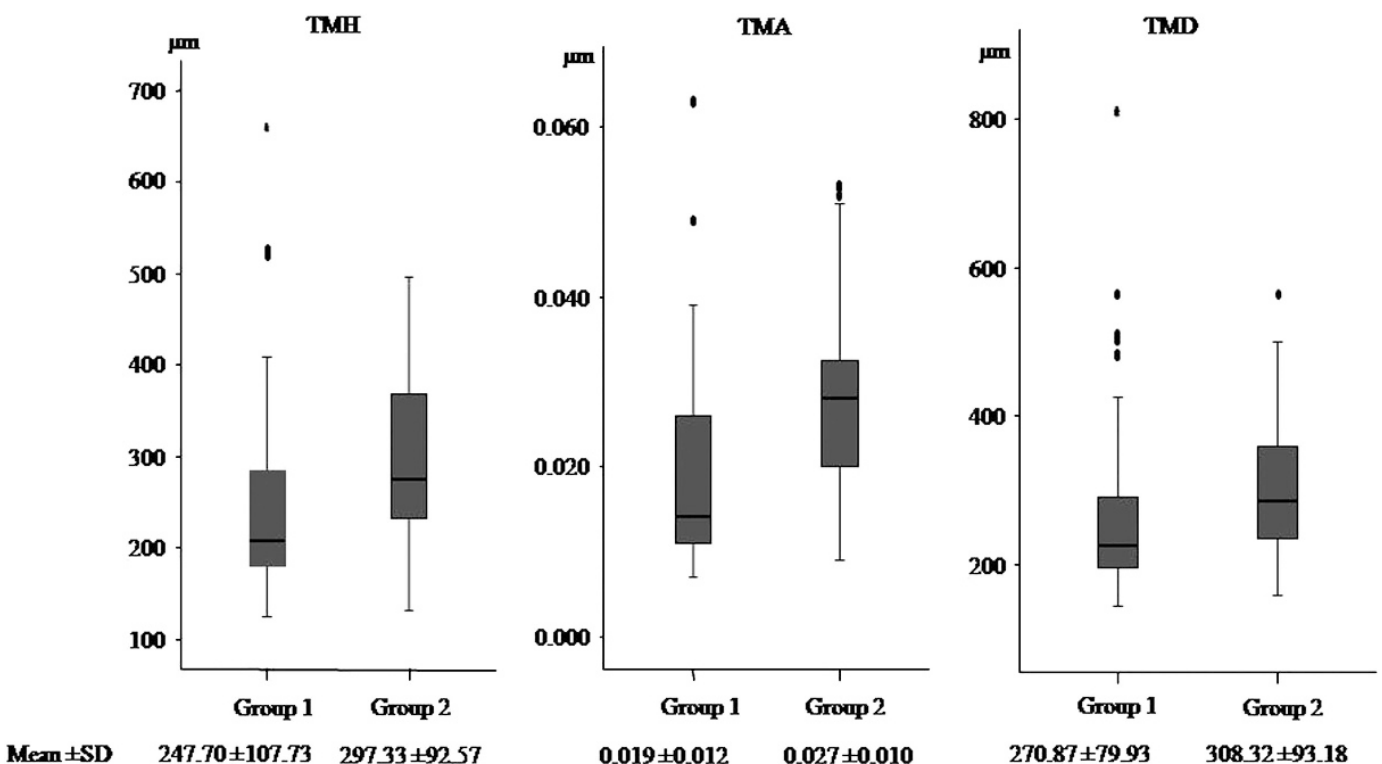

Figure 3 Receiver operating characteristic (ROC) graph analysis delineating the sensitivity and specificity of tear meniscus height (TMH), tear meniscus depth (TMD) and tear meniscus cross-sectional area (TMA) measurements by Fourier-domain optical coherence tomography.

correlations with conventional tests. 12,14,17,18,24,27-30 Moreover, in several studies, the authors also reported that OCT has been good diagnostic sensitivity and specificity in the patients with non-Sjögren's aqueous tear deficiency (ADT), Sjögren's syndrome and lipid tear deficiency. $13,15,16,36$ There are numerous majority of published results on measuring the tear meniscus using the conventional time domain OCT in the literature. ${ }^{11,16,34,35,37}$ But, FD-OCT permits faster image acquisition with significantly higher axial resolution than commercial time domain OCT systems and superior imaging and measurement of tear meniscus. ${ }^{13}$ RTVue FD-OCT acquires 26000 axial scans per second, which is faster than any type of time domain OCT devices. Optovue released a commercially available FD-OCT capable of imaging the anterior segment. It uses two lens attachments, the CAM-S and CAM-long (CAM-L) to obtain anterior segment images. The CAM-S attachment provides a high-magnification view, while the CAM-L attachment provides a wider-viewing angle but at a 
slightly decreased resolution. The RTVue-CAM has a telecentric geometry to reduce image distortion in anterior segment imaging. Moreover, the RTVue-CAM also has higher depth resolution than the previous OCT systems used for tear meniscus measurements. Zhou et al revealed that the RTVue FD-OCT provide more accurate, precise, and reproducible tear meniscus measurement compared with previously used commercial OCT instruments. ${ }^{34}$ Although several articles on the use of FD-OCT in evaluation of tear meniscus and diagnosis of dry eye have been published, there is a lack of universally accepted normative data that limits its clinical use.

The previous studies revealed that the patients report of dry eye symptoms tend to be more reliable and accurate than conventional test results; in addition results of dry eye clinical tests tend to agree poorly with patientreported symtoms. ${ }^{22-27}$ In the tear studies for acne rosacea, TBUT and Schirmer test values were found to be significantly decreased. ${ }^{5-9}$ Quarterman et al also speculated that short TBUT in the patients with cutaneous rosacea might be indicators of ocular rosacea. ${ }^{6}$ Zengin et al found positive correlation between tear film abnormalities and meibomian gland dysfunction, ${ }^{8}$ however, there was no correlation studies between conventional tests results and patient's symptoms in acne rosacea. In our study, Spearman's correlation analysis of clinical examination and OSDI scores indicated that the tear meniscus parameters showed relatively high negative correlation with the severity of clinical dry eye symptoms (OSDI score) in acne rosacea patients. We also found low negative correlation between OSDI scores and TBUT and Schirmer test.

In this study, we found statistically significant difference, but weak correlation between both TBUT and Schirmer test values and tear parameters $(r=0.353-0.391$, $r=0.390-0.404$, respectively). However, in previous dry eye studies have been already showed various correlation rates between conventional tests and tear parameters. In these studies, the authors described low ${ }^{12,13,15,36,38}$ or moderate ${ }^{17,38,39}$ correlation $(r=0.218-0.450$ and $r=0.510-$ 0.602 , respectively) between TBUT and tear parameters. On the other hand, correlation between Schirmer value and tear parameters were rather variable in a number of studies. Chen et $\mathrm{al}^{36}$ found that Schirmer tests were not correlated with tear parameters, while Nguyen et al ${ }^{39}$ found that tear parameters were significantly high correlated with Schirmer's test. Other studies described low ${ }^{12,16}$ or moderate ${ }^{13,15,38}$ correlation between Schirmer tests and tear parameters. This change may be due to different types of dry eye patients enrolled, sample capacity of studies or with or without topical anesthesia using during tests. General consideration is that, a decreased Schirmer value corresponds to decreased tear meniscus parameters. But sometimes because of irritation, patients may have decreased tear parameters but normal Schirmer values. Tear meniscus measurement using OCT could better reflect the natural state of the tear secretion. Many studies have reported that OCT is a good noninvasive method to image and to measure tear meniscus and is able to distinguish between dry eye and non-dry eye.

Many authors analyzed tear meniscus measurement variability by OCT and demonstrated good reproducibility and low interindividual and intraindividual variability. ${ }^{13,15,33,35}$ In our study, the intraindividual and interindividual ICC revealed high repeatability and reproducibility, which is similar to Zhou's ${ }^{33}$ and Qui's ${ }^{15}$ studies. Previous dry eye studies described that the FD-OCT measures tear meniscus with higher reproducibility than previous OCT devices. Because of good repeatability and reproducibility rates in our study, we think that FD-OCT may also be an useful way to measure tear meniscus in acne rosacea patients.

We found that correct diagnosis of dry eye measured by FD-OCT was $60.4 \%$ for TMH, $58.7 \%$ for TMD, and $64.8 \%$ for TMA. In the various dry eye studies, described diagnostic accuracy rates have changed from 50 to $90 \% .^{13,15,16,36,38}$ In these studies, the authors speculated that FD-OCT had likely more diagnostic accuracy for severe dry eye patients. ${ }^{13,15,16}$ We found that the AUC of TMH, TMA and TMD ranged from 0.60 to 0.66 in our study. According to common grading scale, ${ }^{40}$ in our study, this technique demonstrated moderate diagnostic accuracy in acne rosacea patients for dry eye. In several previous studies, Qui et al reported that AUC showed moderate diagnostic accuracy (AUC ranged from 0.66 to 0.71 ) in ADT patients and good diagnostic accuracy (AUC ranged from 0.96 to 0.97 ) in patients with SS. ${ }^{15}$ Our results were less than Shen's data and similar to Ibrahim's and Qui's results. ${ }^{16,17}$

Tear meniscus variable, such as height (TMH), depth (TMD), and cross-sectional area (TMA), have been reported to be useful in the diagnosis of dry eye. ${ }^{12,13,15,16,33,38}$ Some of these studies revealed that TMA is best indicator among the three tear meniscus parameters, but others speculated that TMH may have a potential diagnosis of dry eye patients. We also found that the TMA showed better diagnostic accuracy than TMH and TMD. Because of tear meniscus cross-sectional area could eliminate some mixed factors, such as removing the interference factor of individual palpebral aperture, TMA may provides valuable diagnostic information to clinician with respect to the overall tear volume in the diagnosis of dry eye. ${ }^{13,15}$

In this study, we aimed to search for a new, reliable, and noninvasive method to diagnose dry eye in acne rosacea patients. Tear meniscus parameters are lower in all acne rosacea patients than healthy subjects. Moreover, 
our results suggest acceptable specificity and sensitivity of OCT measurements in diagnosis of dry eye in acne rosacea patients. The present study provides only preliminary results because dry eye in acne rosacea have multifactorial etiology, and further research will be needed to ascertain the dry eye with systemic disease that is best diagnosed with FD-OCT. Although FD-OCT is not considered the gold standard for diagnosis of dry eyes, it is expect to become a valuable diagnostic method for dry eye diagnosis in the future.

\section{Summary}

What was known before

- There is no universally accepted gold standard test for dry eye diagnosis.

- There was a significant correlation between tear meniscus parameters and conventional dry eye tests.

- Measurement of tear mensicus by using anterior segment OCT has potential for diagnosis and monitoring in different types of dry eye including acne rosacea.

- In our study, tear mensicus values were significantly correlated with conventional dry eye tests.

\section{What this study adds}

- Tear meniscus measurement with anterior segment OCT could provide acceptable specificity and sensitivity in diagnosis of dry eye in acne rosacea patients.

\section{Conflict of interest}

The authors declare no conflict of interest.

\section{References}

1 Crawford GH, Pelle MT, James WD. Rosacea: I. Etiology, pathogenesis, and subtype classification. J Am Acad Dermatol 2004; 51: 327-341.

2 Jenkins MS, Brown SI, Lempert SL, Weinberg RJ. Ocular rosacea. Am J Ophthalmol 1979; 88: 618-622.

3 Ghanem VC, Mehra N, Wong S, Mannis MJ. The prevalence of ocular sing in acne rosacea:comparing ophthalmology and dermatology clinics. Cornea 2003; 22: 230-233.

4 Akpek EK, Merchant A, Pinar V, Foster CS. Ocular rosacea: patient characteristics and follow-up. Ophthalmology 1997; 104: 1863-1867.

5 Browning DJ, Proia AD. Ocular rosacea. Surv Ophthalmol 1986; 31: 145-158.

6 Quarterman MJ, Johnson DW, Abele DC, Lesher Jr JL, Hull DS, Davis LS. Ocular rosacea. Sing, symptoms, and tear studies before and after treatment with doxycycline. Arch Dermatol 1997; 133(1): 49-54.

7 Lemp MA, Mahmood MA, Weiler HH. Association of rosacea and keratoconjunctivitis sicca. Arch Ophthalmol 1984; 102(4): 556-557.

8 Zengin N, Tol H, Gunduz K, Okudan S, Balevi S, Endoğru H. Meibomian gland dysfunction and tear film abnormalities in rosacea. Cornea 1995; 14(2): 144-146.
9 Gudmundsen KJ, O’Donnell BF, Powell FC. Schirmer testing for dry eyes in patients with rosacea. J Am Acad Dermatol 1992; 26(2): 211-214.

10 Wolffsohn JS, Peterson RC. Anterior ophthalmic imaging. Clin Exp Optom 2006; 89: 205-214.

11 Savini G, Barboni P, Zanini M. Tear meniscus evaluation by optical coherence tomography. Ophthalmic Surg Lasers Imaging 2006; 37: 112-118.

12 Wang J, Palakuru JR, Aquavella JV. Correlation among upper and lower tear menisci, non-invasive tear break-up time and Schirmer test. Am J Ophthalmol 2008; 145(5): 795-800.

13 Qui X, Gong L, Sun X, Jin H. Age-related variations of human tear meniscus and diagnosis of dry eye with Fourier-domain anterior segment optical coherence tomography. Cornea 2011; 30: 543-549.

14 Mainstone JC, Bruce AS, Golding TR. Tear meniscius mesurement in the diagnosis of dry eye. Curr Eye Res 1996; 15: 653-661.

15 Qui X, Gong L, Lu Y, Jin H, Robitaille M. The diagnostic significance of Fourier-domain optical coherence tomography in Sjögren syndrome, aqueous tear deficiency and lipid tear deficiency patients. Acta Ophthalmol 2012; 90: e359-e366.

16 Shen M, Li J, Wang J. Upper and lower tear menisci in the diagnosis of dry eye. Invest Ophthalmol Vis Sci 2009; 50: 2722-2726.

17 Ibrahim OM, Dogru M, Takano Y, Satake Y, Wakamatsu TH, Fukagawa $\mathrm{K}$ et al. Application of visante optical coherence tomography tear meniscus height measurement in the diagnosis of dry eye disease. Ophthalmology 2010; 117: 1923-1929.

18 Wilkin J, Dahl M, Detmar M, Drake L, Liang MH, Odom $\mathrm{R}$ et al. Standard grading system for rosacea: Report of the National Rosacea Society Expert Committee on the classification and staging of rosacea. J Am Acad Dermatol 2004; 50: 907-912.

19 Irkec M. Turkish OSDI Study Group. Reliability and validity of Turkish translation of the Ocular Surface Disease Index (OSDI) in dry eye syndrome. Invest Ophthalmol Vis Sci 2007; 48: 408.

20 Schiffman RM, Christianson MD, Jacobsen G, Hirsch JD, Reis BL. Reliability and validity of the Ocular Surface Disease Index. Arch Ophthalmol 2000; 118: 615-621.

21 Korb D. Survey of preferred tests for diagnosis of the tear film and dry eye. Cornea 2000; 19: 483-486.

22 Nichols KK, Nichols JJ, Zadnik K. Frequency of dry eye diagnostic test procedures used in various modes of opthalmic practice. Cornea 2000; 19: 477-482.

23 Anonymous. Methodologies to diagnose and monitoring dry eye disease: report of the Diagnostic Methodolgy Subcomittee of the International Dry Eye WorkShop (2007). Ocul Surf 2007; 5: 108-152.

24 Nichols KK, Mitchell GL, Zadnik K. The repeatability of clinical measurements of dry eye. Cornea 2004; 23: 272-285.

25 Yokoi N, Kumuro A. Non-invasive methods of assessing the tear film. Exp Eye Res 2004; 78: 399-407.

26 Nichols KK, Nichols JJ, Mitchell GL. The lack of association between sing and symptoms in patients with dry eye disease. Cornea 2004; 23: 762-770.

27 Lin PY, Cheng CY, Hsu WM, Tsai SY, Lin MW, Liu JH et al. Association between symptoms and sings of dry eye among an elderly Chinese population in Taiwan: the Shihpai Eye Study. Invest Ophthalmol Vis Sci 2005; 46: 1593-1598. 
28 Doughty MJ, Laiquzzaman M, Button NF. Video-assessment of tear meniscus height in elderly Caucasians and its relationship to the exposed ocular surface. Curr Eye Res 2001; 22: $420-426$.

29 Oguz H, Yokoi N, Kinoshita S. The height and radius of the tear meniscus and methods for examinig paramaters. Cornea 2000; 19: 497-500.

30 Uchida A, Uchino M, Goto E, Hosaka E, Kasuya Y, Fukagawa $\mathrm{K}$ et al. Noninvasive interference tear meniscometry in dry eye patients with Sjögren syndrome. Am J Ophthalmol 2007; 144: 232-237.

31 Shen M, Wang J, Tao A, Chen Q, Lin S, Qu J et al. Diurnal variation of upper and lower tear menisci. Am J Ophthalmol 2008; 145: 801-806.

32 Wang J, Aquavella J, Palakuru J, Chung S. Repeated measurements of dynamic tear distribution on the ocular surface after instillation of artificial tears. Invest Ophthalmol Vis Sci 2006; 47: 3325-3329.

33 Zhou S, Li Y, Lu AT, Liu P, Tang M, Yiu SC et al. Reproducibility of tear meniscus measurement by Fourierdomain optical coherence tomography: a pilot study. Ophthalmic Surg Lasers Imaging 2009; 40: 442-447.
34 Keech A, Flanagan J, Simpson, Jones L. Tear meniscus height determination using the OCT2 and the RTVue-100. Optom Vis Sci 2009; 86: 1154-1159.

35 Bitton E, Keech A, Simpson T, Jones L. Variability of the analysis of the tear meniscus height by optical coherence tomography. Optom Vis Sci 2007; 84: 903-908.

36 Chen Q, Zhang X, Cui L, Huang Q, Chen W, Ma H et al. Upper and lower tear menisci in Sjögren's syndrome dry eye. Invest Ophthalmol Vis Sci. 2011; 52(13): 9373-9378.

37 Savini G, Goto E, Carbonelli M, Barboni P, Huang D. Agreement between stratus and visante optical coherence tomography systems in tear meniscus measurements. Cornea 2009; 28: 148-151.

38 Czajkowski G, Kauzny BJ, Laudencka A, Malukiewicz G, Kaluzny JJ. Tear meniscus measurement by spectral optical coherence tomography. Optom Vis Sci 2012; 89(3): 336-342.

39 Nuguyen P, Huang D, Li Y, Sadda SR, Ramos S, Pappuru RR et al. Correaltion between optical coherence tomographyderived asseements of lowe tear meniscus paramaters and clinical features of dry eye disease. Cornea 2012; 31(6): 680-685.

40 Song HH. Analysis of correlated ROC areas in diagnostic testing. Biometrics 1997; 53: 370-372. 